\title{
Human Insulin Therapy Is Associated With an Increased Risk of Lung Cancer: A Population-Based Retrospective Cohort Study
}

\author{
Chin-Hsiao Tseng 1,2,3* \\ ${ }^{1}$ Department of Internal Medicine, National Taiwan University College of Medicine, Taipei, Taiwan, ${ }^{2}$ Division of Endocrinology \\ and Metabolism, Department of Internal Medicine, National Taiwan University Hospital, Taipei, Taiwan, ${ }^{3}$ Division of \\ Environmental Health and Occupational Medicine, National Health Research Institutes, Zhunan, Taiwan
}

OPEN ACCESS

Edited by:

Albert Lecube,

Catalan Health Institute, Spain

Reviewed by:

Jan Brož,

Charles University, Czechia

Silver Karaireho Bahendeka,

Uganda Martyrs University, Uganda

${ }^{*}$ Correspondence:

Chin-Hsiao Tseng

ccktsh@ms6.hinet.net

Specialty section:

This article was submitted to

Diabetes,

a section of the journal

Frontiers in Endocrinology

Received: 17 April 2019

Accepted: 19 June 2019

Published: 11 July 2019

Citation:

Tseng C-H (2019) Human Insulin

Therapy Is Associated With an Increased Risk of Lung Cancer: A Population-Based Retrospective

Cohort Study.

Front. Endocrinol. 10:443. doi: 10.3389/fendo.2019.00443
Background: Whether human insulin may affect lung cancer risk requires investigation.

Methods: All patients with a diagnosis of diabetes mellitus from 1996 to 2009 were enrolled from Taiwan's National Health Insurance. An entry date was set on January 1, 2004, and 1,007,617 patients with type 2 diabetes mellitus diagnosed before 2004 were followed up for new-onset lung cancer until December 31, 2009. Incidence rates of lung cancer for never-users, ever-users, and tertiles of three dose-response exposure parameters (i.e., time since starting insulin, cumulative dose, and cumulative duration) were calculated. Adjusted hazard ratios were estimated by Cox proportional hazards models. The joint effect of insulin and chronic obstructive pulmonary disease was also evaluated.

Results: There were 156,720 ever-users and 850,897 never-users. The respective case numbers of incident lung cancer were 3,007 (1.92\%) and 13,677 (1.61\%), and the respective incidence rates were 424.45 and 313.60 per 100,000 person-years. The adjusted hazard ratio comparing ever-users vs. never-users was 1.545 (95\% confidence interval: 1.478-1.614). The hazard ratios for the different subgroups of the three dose-response parameters all suggested a significantly higher risk of lung cancer associated with insulin use $(P$ trend $<0.0001)$. Compared to patients without insulin use and without chronic obstructive pulmonary disease, insulin users who also had chronic obstructive pulmonary disease had the highest risk of lung cancer (adjusted hazard ratio: 1.891, 95\% confidence interval: 1.767-2.024).

Conclusions: This study suggests a significant association between human insulin use and lung cancer risk in patients with type 2 diabetes mellitus.

Keywords: retrospective cohort study, insulin, lung cancer, type 2 diabetes mellitus, Taiwan

\section{INTRODUCTION}

The insulin-like growth factor (IGF) system, involving two hormones of IGF-1 and IGF-2, two receptors to these two hormones (IGF-1R and IGF-2R), and several binding proteins (IGFBP1-7), plays an important role in the development of lung cancer $(1,2)$. Normally, IGF-1 and IGF-2 are produced by the liver, and they share sequence homology with insulin, which is produced by 
the pancreas (1). There are two types of insulin receptors (IRs), i.e., IR-A (fetal isoform) and IR-B (mature isoform) (2). While IR$B$ is mainly involved in glucose homeostasis, IR-A activation by insulin or IGF-2 may lead to cell proliferation and tumorigenesis (3). IGF-1 may also bind to IR-A, but to a lesser extent of approximately one-tenth affinity of insulin or IGF-2 (2). Lung cancer cells express receptors for insulin (mainly IR-A) and IGF-1 (2) and insulin can activate IR-A and IGF-1R leading to the proliferation of cancer cells (4). Therefore, hyperinsulinemia resulting from either insulin resistance or prolonged insulin administration for the treatment of diabetes may potentially lead to the development of lung cancer.

In a UK study, insulin therapy with or without oral anti-diabetic drugs was not associated with lung cancer (5). However, another study recruiting postmenopausal women in the Women's Health Initiative study in the USA suggested that diabetes increased the risk of lung cancer (hazard ratio: 1.27, 95\% confidence interval: 1.02-1.59), which was more remarkable among patients treated with insulin (hazard ratio: 1.71, 95\% confidence interval: 1.15-2.53) (6). Two meta-analyses also gave different conclusions. While Wu et al. (7) concluded that insulin was associated with an increased risk of lung cancer in patients with diabetes mellitus (odds ratio: 1.23, 95\% confidence interval: 1.10-1.35). Nie et al. (8) did not find an increased risk of lung cancer associated with insulin use (odds ratio: 1.13, 95\% confidence interval: 0.79-1.62).

Because insulin is widely used for the treatment of hyperglycemia in patients with either type 1 or type 2 diabetes mellitus, it is urgently needed to clarify its potential role in lung cancer. Therefore, the present study aimed at investigating whether human insulin use would affect lung cancer risk in Taiwanese patients with type 2 diabetes mellitus.

\section{MATERIALS AND METHODS}

This is a population-based retrospective cohort study that used the reimbursement database of the Taiwan's National Health Insurance (NHI). The study was approved by an ethics review board of the National Health Research Institutes with registered approval number 99274. The database has been de-identified for the protection of privacy and informed consent was not required according to local regulations.

The NHI is a unique and universal healthcare system implemented in Taiwan since March 1995. It is compulsory and covers more than $99.6 \%$ of Taiwan's population. The Bureau of the NHI has contracts with all in-hospitals and approximately 93\% of all medical settings nationwide. The database keeps records of all disease diagnoses, drug prescriptions, and clinical procedures of all insurants for each outpatient visit, use of emergency service, and hospital admission.

During the study period, diabetes mellitus was coded 250.1-250.9 and lung cancer was coded 162, based on the International Classification of Diseases, Ninth Revision, Clinical Modification (ICD-9-CM).

Figure 1 shows the procedures used in selecting patients into the study. At first, an entry date of January 1, 2004, was selected and the database of all patients who had a diagnosis of diabetes mellitus and under treatment with anti-diabetic drugs during the period of 1996-2009 and remained in the insurance program after the entry date were retrieved $(n=1,554,800)$. The entry date was so selected because of the following reasons. First, insulin analogs, which may have a different profile of cancer risk from human insulin $(9,10)$, were not marketed in Taiwan until after February 2004 (the first marketed was insulin glargine). Therefore, all patients enrolled into the study might not have taken any form of insulin analogs before the entry date. Second, because the information of each patient could be tracked from the time of his/her diabetes diagnosis to December 31, 2009, this entry date allowed a maximal follow-up duration of 6 years, such that enough case number of incident lung cancer and enough follow-up duration could be obtained. Third, because insulin analogs might have replaced human insulin originally used by the patients, an entry date set after the introduction of insulin analogs might have made the forms of insulin used by the patients at the time of their enrollment more complicated.

Several criteria were then applied to exclude ineligible patients, such as those who had a diabetes diagnosis after the entry date of January 1, $2004(n=533,525)$, patients with type 1 diabetes mellitus ( $n=5,521$; patients with type 1 diabetes mellitus in Taiwan were issued a so-called "Severe Morbidity Card" after certified diagnosis and they were waived for much of the copayments), duplicated identification number $(n=64)$, unclear information on the date of birth or sex $(n=2,101)$, and patients having a diagnosis of lung cancer before entry date $(n=12,418)$. Finally, 1,007,617 patients were enrolled. These patients had a diagnosis of type 2 diabetes mellitus and under treatment with oral anti-diabetic drugs or human insulin and had no prevalent lung cancer at the time of enrollment.

Patients who had been prescribed insulin (only human insulin was available) before the entry date were defined as ever-users ( $n$ $=156,720,15.55 \%)$. Never-users of insulin $(n=850,897,84.45 \%)$ were defined as patients who had not received any insulin treatment before the entry date. A dose-response relationship was evaluated by using the tertile cutoffs of three variables: time since starting insulin in months, duration of insulin therapy in months, and cumulative dose of insulin in units.

All comorbidities and covariates were determined as a status/diagnosis before the entry date. The ICD-9-CM codes for the comorbidities were as follows: nephropathy 580-589; urinary tract disease 590-599; hypertension 401-405; COPD 490-496; stroke 430-438; ischemic heart disease 410-414; peripheral arterial disease 250.7, 785.4, 443.81, and 440-448; eye disease $250.5,362.0,369,366.41$, and 365.44 ; dyslipidemia 272.0-272.4; congestive heart failure 398.91, 402.11, 402.91, 404.11, 404.13, 404.91, 404.93, and 428; and cancer other than lung cancer 140-208 (excluding 162). Medications included pioglitazone, rosiglitazone, sulfonylurea, meglitinide, metformin, acarbose, statin, fibrate, angiotensin-converting enzyme inhibitor and/or angiotensin receptor blocker, calcium channel blocker, aspirin, and non-steroidal anti-inflammatory drugs. Because the frequency of outpatient visits might have affected the detection rate of lung cancer, the average times of outpatient visits per year were calculated and considered as a potential confounder. 


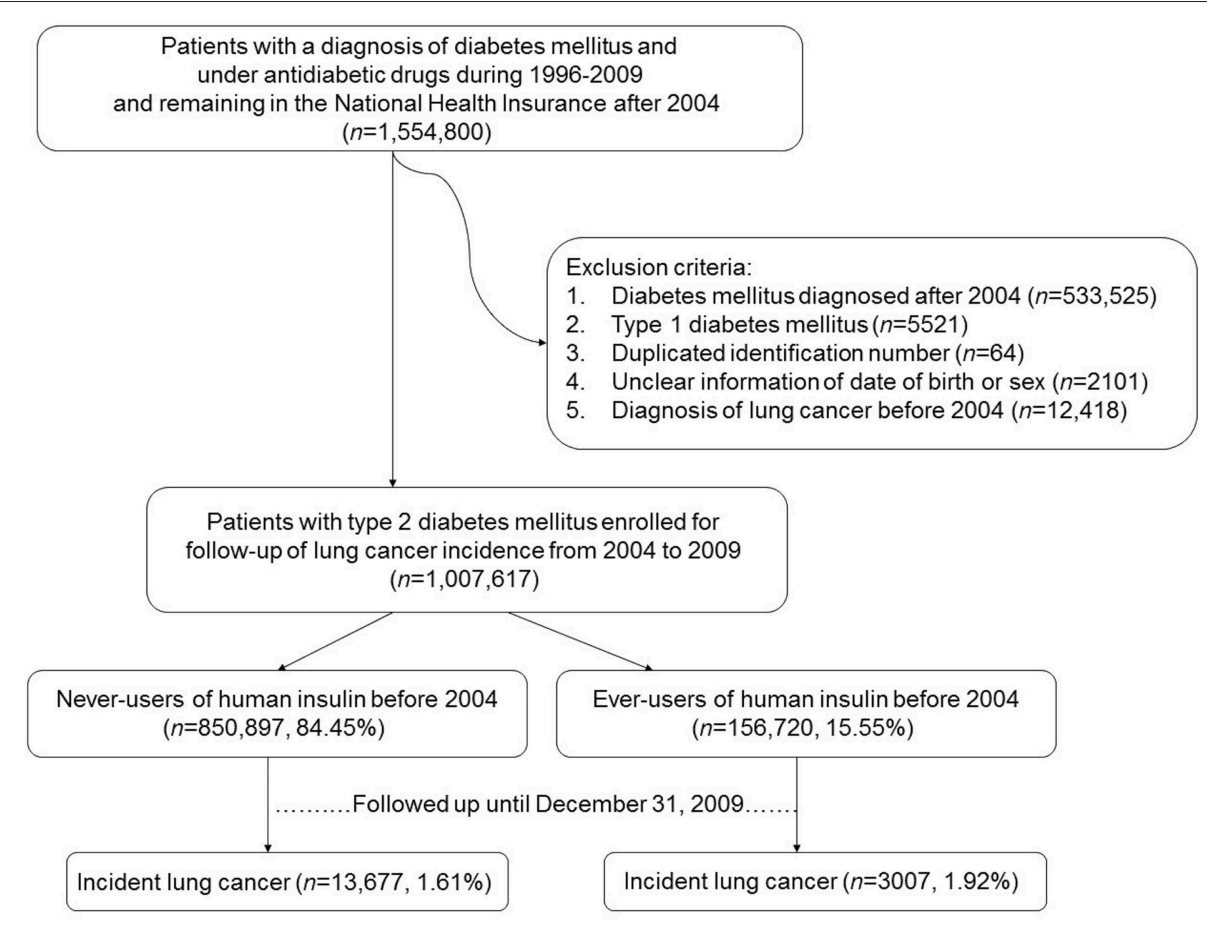

FIGURE 1 | Flowchart showing the procedures used in selecting the studied patients from Taiwan's National Health Insurance database.

Baseline characteristics between ever-users and never-users of insulin were compared by Student's $t$-test for the times of outpatient visits/year and by chi-square test for all other categorical variables.

The incidence density of lung cancer was calculated for neverusers, ever-users, and subgroups of the dose-response parameters of exposure to human insulin. The numerator of the incidence was the number of patients with incident lung cancer during a 6-year follow-up from January 1, 2004, to December 31, 2009. The denominator of the incidence was the person-years of followup. For ever-users, the follow-up duration was censored at lung cancer diagnosis, at the date of the last reimbursement record, or at the date of the initiation of insulin analogs, whichever occurred first. For never-users, the follow-up was censored at the date of lung cancer diagnosis, the last reimbursement record, or any type of insulin (either human insulin or insulin analogs) initiation, whichever occurred first. This ensured no exposure to insulin analogs in the group of ever-users of human insulin throughout the follow-up period, and no exposure to insulin of any type in the referent group of never-users during follow-up.

Cox proportional hazards regression was used to estimate the hazard ratios after adjustment for all variables compared previously as baseline characteristics. $P$ trend was also calculated for the dose-response parameters.

Because COPD is a risk factor of lung cancer (11), to further explore the joint effect between insulin and COPD on lung cancer risk, adjusted hazard ratios were estimated for the following four subgroups: (1) insulin (-)/COPD (-) as referent group, (2) insulin (+)/COPD (-), (3) insulin (-)/COPD (+), and (4) insulin $(+) / \operatorname{COPD}(+)$.
To further exclude the potential residual confounding from COPD, the incidence rates of lung cancer in never-users and everusers of insulin and the adjusted hazard ratios for ever-users vs. never-users of insulin were estimated in subgroups of patients with and without COPD, respectively. $P$ interaction between insulin and COPD was also estimated.

Analyses were conducted using SAS statistical software, version 9.1 (SAS Institute, Cary, NC). $P<0.05$ was considered statistically significant.

\section{RESULTS}

Table 1 shows the baseline characteristics in ever-users $(n=$ $156,720)$ and never-users $(n=850,897)$ of human insulin. All variables differed significantly between the two groups. Everusers are characterized by older age distribution, less male sex, higher proportion with a diabetes duration $\geq 5$ years, higher proportions of all comorbidities and other cancer, higher proportions of using other medications, and more frequent outpatient visits/year.

Table 2 shows the incidence rates of lung cancer in neverusers, ever-users, and users categorized in accordance to the tertiles of the dose-response parameters. The adjusted hazard ratio for ever-users vs. never-users was $1.545(95 \%$ confidence interval: 1.478-1.614), suggesting a significantly higher risk of lung cancer associated with human insulin use. In the models evaluating the dose-response parameters, hazard ratios were significant in all subgroups with $P$ trend $<$ 0.0001 . The hazard ratios ( $95 \%$ confidence intervals) were also significant for the following covariates enrolled in the model 
TABLE 1 | Baseline characteristics in never-users and ever-users of human insulin.

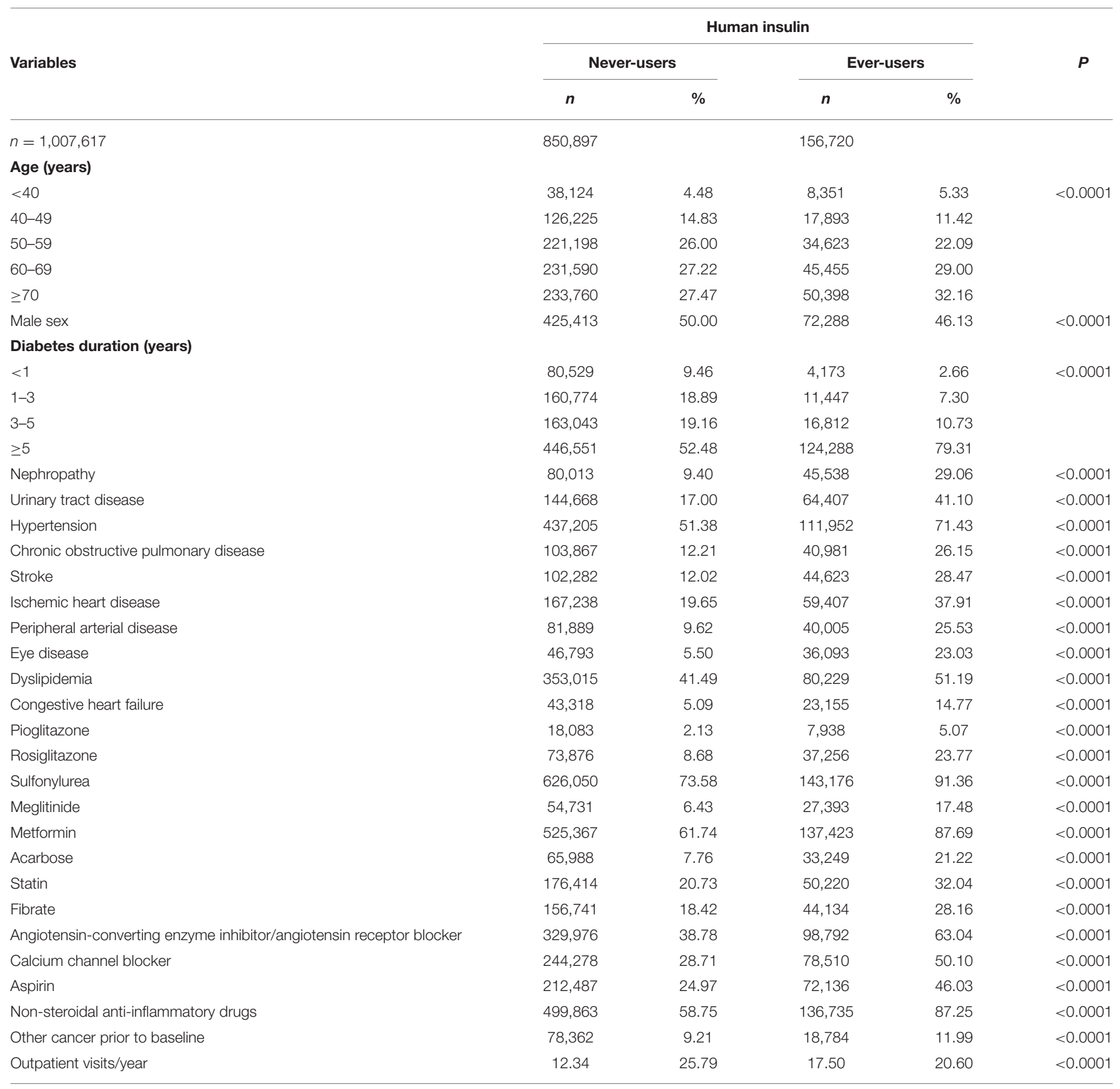

Outpatient visits/year is expressed as mean and standard deviation.

evaluating ever-users vs. never-users of insulin: age [2.460 (2.020-2.995), 4.279 (3.540-5.172), 8.355 (6.923-10.083), and 14.409 (11.941-17.387) for $40-49,50-59,60-69$, and $\geqq 70$ years vs. $<40$ years, respectively], sex [men vs. women: 1.847 (1.7891.907)], hypertension [0.884 (0.849-0.922)], COPD [1.413 $(1.359-1.469)]$, eye disease [0.921 (0.869-0.976)], dyslipidemia [0.914 (0.882-0.948)], congestive heart failure [1.074 (1.011$1.140)]$, other cancer prior to baseline [1.593 (1.527-1.662)], metformin $[0.952(0.916-0.990)]$, acarbose $[0.901 \quad(0.850-$ $0.954)]$, statin [0.935 (0.895-0.976)], and angiotensin-converting enzyme inhibitor and/or angiotensin receptor blocker [0.892 (0.856-0.929)].

Table 3 shows the joint effects of insulin and COPD on the risk of lung cancer. Compared to the referent subgroup of insulin (-)/COPD (-), all the other three subgroups showed a significantly higher risk of lung cancer. Patients who had COPD and were using insulin had the highest risk, with an estimated hazard ratio of 1.891 (95\% confidence interval: 1.767-2.024).

Table 4 shows the subgroup analyses in patients with and without COPD. A significantly higher risk of lung cancer 
TABLE 2 | Exposure to human insulin and incidences of lung cancer and hazard ratios comparing exposed to unexposed.

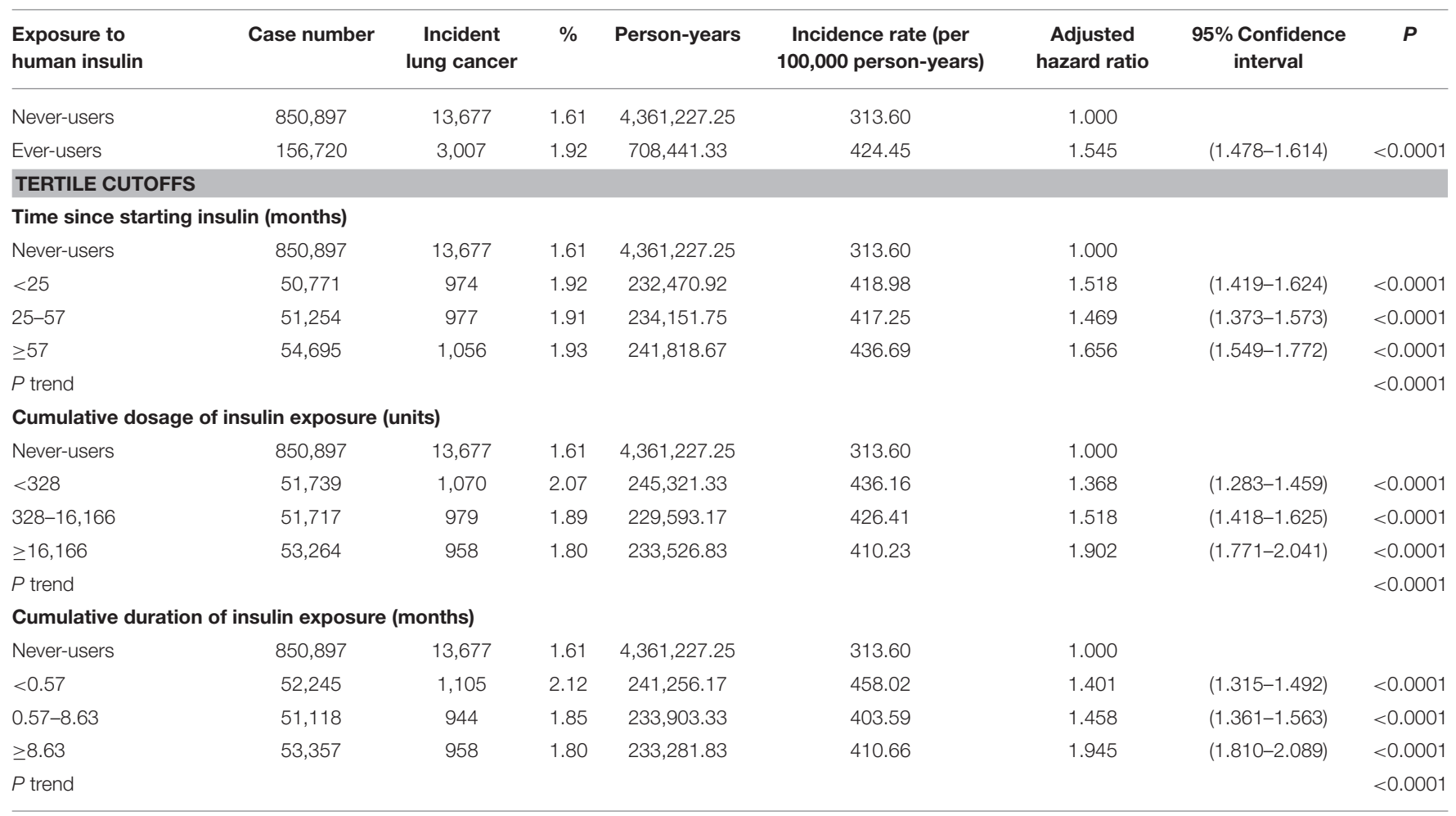

Hazard ratios are adjusted for all variables in Table 1.

TABLE 3 | Joint effects of human insulin and chronic obstructive pulmonary disease on lung cancer.

\begin{tabular}{|c|c|c|c|c|c|c|}
\hline Human insulin & COPD & Number of incident lung cancer & Number of cases followed & Adjusted hazard ratio & 95\% Confidence interval & $P$ \\
\hline No & No & 11,064 & 747,030 & 1.000 & & \\
\hline Yes & No & 1,937 & 115,739 & 1.383 & $(1.313-1.456)$ & $<0.0001$ \\
\hline No & Yes & 2,613 & 103,867 & 1.429 & $(1.367-1.494)$ & $<0.0001$ \\
\hline Yes & Yes & 1,070 & 40,981 & 1.891 & $(1.767-2.024)$ & $<0.0001$ \\
\hline
\end{tabular}

Hazard ratios are adjusted for all variables in Table 1. COPD, chronic obstructive pulmonary disease.

associated with insulin use was consistently observed, and no interaction between insulin and COPD was noted.

\section{DISCUSSION}

The findings of the present study suggested that human insulin use in patients with type 2 diabetes mellitus was associated with an increased risk of lung cancer in a dose-response pattern (Table 2). Furthermore, insulin and COPD might jointly enhance the development of lung cancer (Table 3).

The mechanisms of insulin-related lung cancer risk remain to be investigated. Some basic research provides possible explanations. An in vitro study using non-small-cell lung cancer cells suggested that insulin promotes the proliferation, migration, and invasion of lung cancer by increasing the phosphorylation of IR substrate 1 and activating the phosphoinositide 3kinase/protein kinase B pathway (12). Another study showed that ablation of IR substrates 1 and 2 suppressed Kras-driven lung tumorigenesis (13). Because lung cancer cells may overexpress IR-A and IGF-1R, the binding of insulin to these receptors triggers the mitogenic pathways (2). Furthermore, because IGF2 has high affinity to IR-A (2), the IGF-2 produced by cancer cells may exert an autocrine/paracrine effect by binding to IRA and promote cancer cell proliferation (2). A study conducted in the Chinese population showed that IGF-1R was highly expressed in type 2 diabetes patients with lung cancer (14). Type 2 diabetes mellitus is characterized by insulin resistance in the metabolic pathway involving IR-B, with compensatory increase in the secretion of insulin (hyperinsulinemia). The compensatory hyperinsulinemia may overstimulate the mitogenic pathway and promote cell proliferation via IR-A or IGF-1R (2). Similarly, subcutaneous insulin injection for the treatment of hyperglycemia in patients with type 2 diabetes mellitus may cause hyperinsulinemia, especially in the presence of insulin resistance, which, in turn, may stimulate the mitogenic pathways involving IR-A and IGF-1R leading to new cancer development or may 
TABLE 4 | Subgroup analyses in patients with and without chronic obstructive pulmonary disease.

\begin{tabular}{|c|c|c|c|c|c|c|c|c|}
\hline $\begin{array}{l}\text { Exposure to } \\
\text { human insulin }\end{array}$ & $\begin{array}{l}\text { Case } \\
\text { number }\end{array}$ & $\begin{array}{l}\text { Incident lung } \\
\text { cancer }\end{array}$ & $\%$ & Person-years & $\begin{array}{l}\text { Incidence rate (per 100,000 } \\
\text { person-years) }\end{array}$ & $\begin{array}{c}\text { Adjusted } \\
\text { hazard ratio }\end{array}$ & $\begin{array}{l}95 \% \text { Confidence } \\
\text { interval }\end{array}$ & $P$ \\
\hline \multicolumn{9}{|c|}{ PATIENTS WITH COPD } \\
\hline Ever-users & 40,981 & 1,070 & 2.61 & $172,564.33$ & 620.06 & 1.546 & $(1.430-1.673)$ & $<0.0001$ \\
\hline \multicolumn{9}{|c|}{ PATIENTS WITHOUT COPD } \\
\hline
\end{tabular}

Hazard ratios are adjusted for all variables in Table 1. COPD, chronic obstructive pulmonary disease. P interaction between insulin and COPD =0.2035.

accelerate cell proliferation in the existence of occult lung cancer cells (2).

There are some clinical implications with these findings. First, because human insulin remains widely used for the treatment of hyperglycemia in either type 1 or type 2 diabetes patients, its potential risk of lung cancer warrants intensive investigation and immediate confirmation. Because the use of human insulin through inhalation has been shown to increase the risk of lung cancer (15), the prolonged use of insulin via subcutaneous injection is highly possible. Second, because this study investigated only the effect of human insulin, the findings could not be readily generalized to insulin analogs. However, because insulin glargine, the most commonly used insulin analog in Taiwan, has a higher affinity to IGF-1R (9), its potential carcinogenic effect on the lung should be highly suspected. Before human insulin, insulin glargine or other types of insulin analogs can be proven to be safe from lung carcinogenesis; the longterm use of insulin to treat diabetes should better be reserved for patients whose hyperglycemia cannot be satisfactorily treated with other anti-diabetic drugs and who are not having significant risk factors of lung cancer such as COPD as shown in the present study (Table 3). Third, the oral delivery of insulin under development for clinical use requires more intensive and cautious evaluation of cancer risk, especially of cancer involving the gastrointestinal tracts.

The present study has several strengths. First, because the NHI covers nearly the whole population in Taiwan and the longitudinal data of the patients were available throughout the period since the patients were diagnosed with diabetes until the end of 2009, the findings can be readily generalized to the whole population. Second, recall bias from self-reporting, a problem always associated with a retrospective study design, could be avoided by using the medical records. Third, detection bias in association with different socioeconomical status is less likely in this study because the NHI considers cancer as a severe morbidity and most medical co-payments can be waived. Furthermore, the drug cost-sharing in the NHI system is relatively low and can be waived in patients with low income, veterans, or those receiving prescription refills for chronic disease.

One of the major limitations of the study is the lack of information on cigarette smoking and only COPD could be used as a surrogate marker. Because the prevalence rate of
COPD in ever-users of insulin was significantly higher than that in never-users of insulin (26.16 vs. $12.21 \%$, Table 1 ), the subgroup analyses (Table 4) suggested that COPD might not exert a potential residual confounding effect. Furthermore, because COPD could only be partly interpreted as an indirect marker of smoking, there remained a possible confounding effect of smoking in the study if ever-users of insulin did have a significantly higher smoking rate than never-users of insulin. There was one large epidemiological study comparing the smoking rates in the Taiwanese diabetes patients during the same period (cohort enrolled with questionnaire interview from 1995 to 2002). It was noted that the prevalence rate of smoking among users of insulin was in fact lower than non-users of insulin in patients with type 2 diabetes mellitus in Taiwan. The study compared 81,923 non-users of insulin and 5,927 users of insulin and showed that the prevalence rates of smoking were $30.7 \%$ and $27.9 \%(P<0.001)$, respectively $(16)$. Therefore, if smoking played a confounding effect in the present study, this might only have underestimated the hazard ratio of lung cancer associated with insulin use.

Another limitation of the study is the lack of information on radon exposure because it is the second most important risk factor of lung cancer in the USA $(17,18)$. Previous studies in Taiwan suggested that radon concentrations may be high in spa water (19) and in indoor environment during cool season because of low ventilation rates (20). Therefore, inhabitants living near the source of spa water, patients who always took the spa, or patients who stayed mainly indoors during the cold season might potentially have an increased risk of lung cancer. Although studies are still lacking with regard to lung cancer risk related to radon exposure in Taiwan, the confounding effect of radon exposure should be clarified in future studies. However, because a confounder must be simultaneously associated with exposure and outcome and must not be part of the causal pathway (21), if radon exposure is not correlated with insulin use, its potential confounding in the present study may be minimal.

A third limitation is associated with the use of the reimbursement database. Many different healthcare professionals were involved in patient care, and the measurement/diagnosis of risk factors and outcome throughout the database might not be accurate and consistent compared to that achieved with a prospective cohort study. There is also a possibility that the association between risk factors and lung cancer 
would change with time and the presence of incomplete records might have biased the observation. This may be particularly so if the data were not missing at random (i.e., if missing data were related to the exposure of insulin use or the outcome of lung cancer). Therefore, only association and not causation can be inferred from the present study.

Other limitations included a lack of actual measurement data for confounders such as alcohol drinking, other occupational and environmental exposure, family history, exercise, lifestyle, diet, biochemical and hormonal data, genetic parameters, as well as the lack of information on the pathology, grading, and staging of lung cancer.

In conclusion, this population-based retrospective cohort study in Taiwan suggests an association between human insulin use and lung cancer. However, this should better be confirmed by additional studies in other ethnicities or by using a clinical trial. Whether insulin glargine or other insulin analogs may also increase the risk of lung cancer was not addressed here and is an issue awaiting urgent investigation.

\section{DATA AVAILABILITY}

The datasets for this manuscript are not publicly available because public availability of the dataset is restricted by local regulations to protect privacy. Requests to access the datasets should be directed to C-HT, ccktsh@ms6.hinet.net.

\section{REFERENCES}

1. Cevenini A, Orrù S, Mancini A, Alfieri A, Buono $\mathrm{P}$, Imperlini E. Molecular signatures of the insulin-like growth factor 1-mediated epithelialmesenchymal transition in breast, lung and gastric cancers. Int J Mol Sci. (2018) 19:E2411. doi: 10.3390/ijms19082411

2. Vella V, Milluzzo A, Scalisi NM, Vigneri P, Sciacca L. Insulin receptor isoforms in cancer. Int J Mol Sci. (2018) 19:3615. doi: 10.3390/ijms191 13615

3. Djiogue S, Nwabo Kamdje AH, Vecchio L, Kipanyula MJ, Farahna M, Aldebasi Y, et al. Insulin resistance and cancer: the role of insulin and IGFs. Endocr Relat Cancer. (2013) 20:R1-7. doi: 10.1530/ERC-12-0324

4. Mancarella C, Scotlandi K. IGF system in sarcomas: a crucial pathway with many unknowns to exploit for therapy. J Mol Endocrinol. (2018) 61:T45-60. doi: 10.1530/JME-17-0250

5. Hall GC, Roberts CM, Boulis M, Mo J, MacRae KD. Diabetes and the risk of lung cancer. Diabetes Care. (2005) 28:590-4. doi: 10.2337/diacare.28. 3.590

6. Luo J, Chlebowski R, Wactawski-Wende J, Schlecht NF, Tinker L, Margolis KL. Diabetes and lung cancer among postmenopausal women. Diabetes Care. (2012) 35:1485-91. doi: 10.2337/dc11-2108

7. Wu Y, Liu HB, Shi XF, Song Y. Conventional hypoglycaemic agents and the risk of lung cancer in patients with diabetes: a meta-analysis. PLOS ONE. (2014) 9:e99577. doi: 10.1371/journal.pone.0099577

8. Nie SP, Chen H, Zhuang MQ, Lu M. Anti-diabetic medications do not influence risk of lung cancer in patients with diabetes mellitus: a systematic review and meta-analysis. Asian Pac J Cancer Prev. (2014) 15:6863-9. doi: 10.7314/APJCP.2014.15.16.6863

9. Kurtzhals P, Schäffer L, Sørensen A, Kristensen C, Jonassen I, Schmid C, et al. Correlations of receptor binding and metabolic and mitogenic potencies of insulin analogs designed for clinical

\section{ETHICS STATEMENT}

The study was approved by an ethic review board of the National Health Research Institutes with registered approval number 99274. The database has been de-identified for the protection of privacy and informed consent was not required according to local regulations.

\section{AUTHOR CONTRIBUTIONS}

C-HT researched the data and wrote the manuscript.

\section{FUNDING}

The study was supported by the Ministry of Science and Technology (MOST 107-2221-E-002-129-MY3) of Taiwan. The funder had no role in study design, data collection and analysis, decision to publish, or preparation of the manuscript.

\section{ACKNOWLEDGMENTS}

The study is based in part on data from the National Health Insurance Research Database provided by the Bureau of National Health Insurance, Department of Health and managed by National Health Research Institutes. The interpretation and conclusions contained herein do not represent those of the Bureau of National Health Insurance, Department of Health or National Health Research Institutes. use. Diabetes. (2000) 49:999-1005. doi: 10.2337/diabetes.4
9.6 .999

10. Mannucci E, Monami M, Balzi D, Cresci B, Pala L, Melani C, et al. Doses of insulin and its analogues and cancer occurrence in insulin-treated type 2 diabetic patients. Diabetes Care. (2010) 33:1997-2003. doi: 10.2337/dc 10-0476

11. Houghton AM. Mechanistic links between COPD and lung cancer. Nat Rev Cancer. (2013) 13:233-45. doi: 10.1038/nrc3477

12. Jiang J, Ren HY, Geng GJ, Mi YJ, Liu Y, Li N, et al. Oncogenic activity of insulin in the development of non-small cell lung carcinoma. Oncol Lett. (2018) 15:447-52. doi: 10.3892/ol.2017.7347

13. Xu H, Lee MS, Tsai PY, Adler AS, Curry NL, Challa S, et al. Ablation of insulin receptor substrates 1 and 2 suppresses Kras-driven lung tumorigenesis. Proc Natl Acad Sci USA. (2018) 115:4228-33. doi: 10.1073/pnas.1718 414115

14. Ding J, Tang J, Chen X, Men HT, Luo WX, Du Y, et al. Expression characteristics of proteins of the insulin-like growth factor axis in nonsmall cell lung cancer patients with preexisting type 2 diabetes mellitus. Asian Pac J Cancer Prev. (2013) 14:5675-80. doi: 10.7314/APJCP.2013.14.1 0.5675

15. Kling J. Inhaled insulin's last gasp? Nat Biotechnol. (2008) 26:479-80. doi: $10.1038 /$ nbt0508-479

16. Tseng $\mathrm{CH}$. Exogenous insulin use and hypertension in adult patients with type 2 diabetes mellitus. Arch Intern Med. (2006) 166:1184-9. doi: 10.1001/archinte.166.11.1184

17. de Groot PM, Wu CC, Carter BW, Munden RF. The epidemiology of lung cancer. Transl Lung Cancer Res. (2018) 7:220-33. doi: $10.21037 /$ tlcr.2018.05.06

18. Vogeltanz-Holm N, Schwartz GG. Radon and lung cancer: what does the public really know? J Environ Radioact. (2018) 192:26-31. doi: 10.1016/j.jenvrad.2018.05.017 
19. Weng PS, Lin CL. Radon concentrations in spa water taken from hot and cold springs in Taiwan. Appl Radiat Isot. (1995) 46:293-5. doi: 10.1016/0969-8043(94)00143-N

20. Iimoto $\mathrm{T}$, Kosako $\mathrm{T}$, Sugiura N. Measurements of summer radon and its progeny concentrations along with environmental gamma dose rates in Taiwan. J Environ Radioact. (2001) 57:57-66. doi: 10.1016/S0265-931X(01)00011-X

21. Gerhard T. Bias: considerations for research practice. Am J Health Syst Pharm. (2008) 65:2159-68. doi: 10.2146/ajhp0 70369
Conflict of Interest Statement: The author declares that the research was conducted in the absence of any commercial or financial relationships that could be construed as a potential conflict of interest.

Copyright (c) 2019 Tseng. This is an open-access article distributed under the terms of the Creative Commons Attribution License (CC BY). The use, distribution or reproduction in other forums is permitted, provided the original author(s) and the copyright owner(s) are credited and that the original publication in this journal is cited, in accordance with accepted academic practice. No use, distribution or reproduction is permitted which does not comply with these terms. 